\title{
New Oscillation Criteria for Third-Order Nonlinear Functional Differential Equations
}

\author{
Quanxin Zhang, ${ }^{1}$ Li Gao, ${ }^{1}$ Shouhua Liu, ${ }^{1}$ and Yuanhong $\mathrm{Yu}^{2}$ \\ ${ }^{1}$ Department of Mathematics, Binzhou University, Shandong 256603, China \\ ${ }^{2}$ Academy of Mathematics and System Sciences, Chinese Academy of Sciences, Beijing 100190, China \\ Correspondence should be addressed to Quanxin Zhang; 3314744@163.com
}

Received 19 November 2013; Accepted 13 January 2014; Published 11 March 2014

Academic Editor: Tongxing Li

Copyright (c) 2014 Quanxin Zhang et al. This is an open access article distributed under the Creative Commons Attribution License, which permits unrestricted use, distribution, and reproduction in any medium, provided the original work is properly cited.

\begin{abstract}
This paper discusses oscillatory and asymptotic behavior of solutions of a class of third-order nonlinear functional differential equations. By using the generalized Riccati transformation and the integral averaging technique, three new sufficient conditions which insure that the solution is oscillatory or converges to zero are established. The results obtained essentially generalize and improve the earlier ones.
\end{abstract}

\section{Introduction}

As is well known, the comparison and separation theory of zeros distribution for second-order homogeneous linear differential equations established by Ladde et al. lays a foundation of oscillation theory for differential equations. During one and a half century, oscillation theory of differential equations has developed quickly and played an important role in qualitative theory of differential equations and the theory of boundary value problem. Oscillation theory of differential equations has been widely used in areas of physics, mechanics, radio technology, control system, sciences of life, economic relations, and population growth. The oscillations are physical phenomena which widely exist in physics and technological sciences, such as the oscillation of building and machine, electromagnetic vibration in radio technology and optical science, self-excited vibration in control system, sound vibration, beam vibration in synchrotron accelerator, the vibration sparked for burning rocket engine, and the complicated oscillation in chemical reaction. All different phenomena can be unified into an oscillation theory through an oscillation equation. There are many books on the oscillation theory, about which we can refer to [1].

The oscillation theory of third-order nonlinear functional differential equations has been widely applied in research of a lossless high-speed computer network and physical sciences.
In this paper, we are concerned with oscillatory behavior of a third-order nonlinear functional differential equation as follows:

$$
\left(r_{2}(t)\left[\left(r_{1}(t) x^{\prime}(t)\right)^{\prime}\right]^{\alpha}\right)^{\prime}+q(t) f(x[g(t)])=0, \quad t \geq t_{0},
$$

where $\alpha$ is the ratio of positive odd integers. We have the following hypotheses:

$\left(\mathrm{A}_{1}\right) r_{1}(t), r_{2}(t), q(t) \in C^{1}\left(\left[t_{0}, \infty\right),(0, \infty)\right)$ and $r_{1}(t), r_{2}(t)$ satisfy

$$
\int_{t_{0}}^{\infty} \frac{1}{r_{1}(s)} \mathrm{d} s=\infty, \quad \int_{t_{0}}^{\infty}\left(\frac{1}{r_{2}(s)}\right)^{1 / \alpha} \mathrm{d} s=\infty ;
$$

$\left(\mathrm{A}_{2}\right) f(u) \in C(R, R)$, such that $f(u) / u^{\alpha} \geq K>0, u \neq 0$;

$\left(\mathrm{A}_{3}\right) g(t) \in C^{1}\left(\left[t_{0}, \infty\right), R\right), g^{\prime}(t) \geq 0$, and $\lim _{t \rightarrow \infty} g(t)=$ $\infty$.

By a solution of (1), we mean a nontrivial function $x(t)$ satisfying (1) which has the properties $x(t) \in C^{1}\left(\left[T_{x}, \infty\right), R\right)$ for $T_{x} \geq t_{0}$ and $r_{2}(t)\left[\left(r_{1}(t) x^{\prime}(t)\right)^{\prime}\right]^{\alpha} \in C^{1}\left(\left[T_{x}, \infty\right), R\right)$. Our attention is paid to those solutions of (1) which satisfy $\sup \{|x(t)|: t \geq T\}>0$ for all $T \geq T_{x}$. A solution $x$ of $(1)$ is said 
to be oscillatory on $\left[T_{x}, \infty\right)$ if it is neither eventually positive nor eventually negative. Otherwise, it is called nonoscillatory. The equation itself is called oscillatory if all its solutions are oscillatory.

In recent years, there have been numerous researches or many research activities concerning the oscillation and nonoscillation of solutions of three-order functional differential equations, which are special cases of (1), and for recent contributions, we refer to [2-8]. Consider

$$
\begin{gathered}
x^{\prime \prime \prime}(t)+q(t) x[g(t)]=0, \quad t \geq t_{0} \\
\left(r_{2}(t)\left[\left(r_{1}(t) x^{\prime}(t)\right)^{\prime}\right]^{\alpha}\right)^{\prime}+q(t) x^{\alpha}(t)=0, \quad t \geq t_{0} ; \\
\left(r_{2}(t)\left(r_{1}(t) x^{\prime}(t)\right)^{\prime}\right)^{\prime}+q(t) f(x[g(t)])=0, \quad t \geq t_{0} \\
\left(r(t)\left[x^{\prime \prime}(t)\right]^{\alpha}\right)^{\prime}+q(t) f(x[g(t)])=0, \quad t \geq t_{0} .
\end{gathered}
$$

Parhi and Padhi [2] studied asymptotic behavior of solutions of (3). By using the integral averaging technique, Baculíková et al. [3] obtained sufficient conditions which insured that the solution of self-liner ordinary differential equation (4) was oscillatory or converges to zero. Mojsej [4] established the comparison results which insured that the solution of (5) was oscillatory or converges to zero. By the integral averaging technique, Saker [5] gave some oscillatory results of (5) when the condition $g(t)=t-\sigma$ holds. Several authors had proved some oscillatory results of (6) by method of comparison; see [6-8]. In this paper we intend to use Riccati transformation and the integral averaging technique to obtain some sufficient conditions which guarantee that every solution $x(t)$ of $(1)$ is oscillatory or converges to zero. Our results generalize and improve the corresponding theorems established in $[3,5]$.

\section{Several Lemmas}

Lemma 1. Assume that $x(t)$ is a positive solution of (1). Then, there exists $T \in\left[t_{0}, \infty\right)$ such that either

(I) $x(t)>0, x^{\prime}(t)>0,\left(r_{1}(t) x^{\prime}(t)\right)^{\prime}>0, t \geq T$,

or

(II) $x(t)>0, x^{\prime}(t)<0,\left(r_{1}(t) x^{\prime}(t)\right)^{\prime}>0, t \geq T$. 1].

The proof is similar to that of [3, Lemma 1] or [7, Lemma

Throughout this paper, for sufficiently large $T$, we denote

$$
\begin{gathered}
R_{1}(t, T)=\int_{T}^{t}\left(\frac{1}{r_{2}(s)}\right)^{1 / \alpha} \mathrm{d} s, \\
R_{2}(t, T)=\int_{T}^{t} \frac{R_{1}(s, T)}{r_{1}(s)} \mathrm{d} s, \\
Q(t)=\left(K \int_{t}^{\infty} q(s) \mathrm{d} s\right)^{1 / \alpha} .
\end{gathered}
$$

In order to make the definition of $Q(t)$ meaningful, we denote

$$
\int_{t}^{\infty} q(s) \mathrm{d} s<\infty .
$$

Lemma 2. Assume that $x(t)$ is a positive solution of (1) which satisfies case (I) in Lemma 1 . Then there exists $T \in\left[t_{0}, \infty\right)$, such that

$$
\begin{gathered}
x^{\prime}(t)>\frac{R_{1}(t, T)}{r_{1}(t)} r_{2}^{1 / \alpha}(t)\left(r_{1}(t) x^{\prime}(t)\right)^{\prime}, \quad t \geq T, \\
x(t)>R_{2}(t, T) r_{2}^{1 / \alpha}(t)\left(r_{1}(t) x^{\prime}(t)\right)^{\prime}, \quad t \geq T .
\end{gathered}
$$

Assume that (8) and $g(t) \geq t$ hold. Then

$$
x(t)<\frac{1}{Q(t)} r_{2}^{1 / \alpha}(t)\left(r_{1}(t) x^{\prime}(t)\right)^{\prime}, \quad t \geq T .
$$

Proof. Pick $T \in\left[t_{0}, \infty\right)$ so that $x[g(t)]>0$ for $t \geq T$. Using (1), we obtain

$$
\begin{aligned}
\left(r_{2}(t)\left[\left(r_{1}(t) x^{\prime}(t)\right)^{\prime}\right]^{\alpha}\right)^{\prime} & =-q(t) f(x[g(t)]) \\
& \leq-K q(t) x^{\alpha}[g(t)]<0, \quad t \geq T .
\end{aligned}
$$

Then, $r_{2}(t)\left[\left(r_{1}(t) x^{\prime}(t)\right)^{\prime}\right]^{\alpha}$ is strictly decreasing on $[T, \infty)$. We get

$$
\begin{aligned}
r_{1}(t) x^{\prime}(t) & >r_{1}(t) x^{\prime}(t)-r_{1}(T) x^{\prime}(T) \\
& =\int_{T}^{t} \frac{\left(r_{2}(s)\left[\left(r_{1}(s) x^{\prime}(s)\right)^{\prime}\right]^{\alpha}\right)^{1 / \alpha}}{r_{2}^{1 / \alpha}(s)} \mathrm{d} s \\
& \geq\left(r_{2}(t)\left[\left(r_{1}(t) x^{\prime}(t)\right)^{\prime}\right]^{\alpha}\right)^{1 / \alpha} \int_{T}^{t}\left(\frac{1}{r_{2}(s)}\right)^{1 / \alpha} \mathrm{d} s,
\end{aligned}
$$

and, hence, we have

$$
x^{\prime}(t)>\frac{R_{1}(t, T)}{r_{1}(t)} r_{2}^{1 / \alpha}(t)\left(r_{1}(t) x^{\prime}(t)\right)^{\prime}, \quad t \geq T .
$$

By integrating both sides of the above inequality from $T$ to $t$, it yields

$$
x(t)>R_{2}(t, T) r_{2}^{1 / \alpha}(t)\left(r_{1}(t) x^{\prime}(t)\right)^{\prime}, \quad t \geq T .
$$

Furthermore, by integrating both sides of (1) from $t$ to $\infty$ and noting that $x^{\prime}(t)>0, g^{\prime}(t) \geq 0, g(t) \geq t$, we obtain

$$
\begin{aligned}
r_{2}(t)\left[\left(r_{1}(t) x^{\prime}(t)\right)^{\prime}\right]^{\alpha} & >\int_{t}^{\infty} q(s) f(x[g(s)]) \mathrm{d} s \\
& \geq K \int_{t}^{\infty} q(s) x^{\alpha}[g(s)] \mathrm{d} s \\
& \geq K \int_{t}^{\infty} q(s) x^{\alpha}(s) \mathrm{d} s \\
& \geq\left(K \int_{t}^{\infty} q(s) \mathrm{d} s\right) x^{\alpha}(t) .
\end{aligned}
$$


Then,

$$
x(t)<\frac{1}{Q(t)} r_{2}^{1 / \alpha}(t)\left(r_{1}(t) x^{\prime}(t)\right)^{\prime} .
$$

This completes the proof.

Lemma 3. Assume that $x(t)$ is a positive solution of (1) which satisfies case (II) in Lemma 1. Furthermore,

$$
\int_{t_{0}}^{\infty} \frac{1}{r_{1}(t)} \int_{t}^{\infty}\left[\frac{1}{r_{2}(s)} \int_{s}^{\infty} q(u) \mathrm{d} u\right]^{1 / \alpha} \mathrm{d} s \mathrm{~d} t=\infty .
$$

Then, $\lim _{t \rightarrow \infty} x(t)=0$.

Proof. Assume that $x(t)$ is a positive solution of (1) which satisfies case (II) in Lemma 1. Then, $x(t)$ is decreasing and $\lim _{t \rightarrow \infty} x(t)=l \geq 0$. We assert that $l=0$. If not, then $x[g(t)] \geq l>0, t \geq T$. Integrating (1) from $t$ to $\infty$, we get

$$
\begin{aligned}
-r_{2}(t)\left[\left(r_{1}(t) x^{\prime}(t)\right)^{\prime}\right]^{\alpha} & \leq-K \int_{t}^{\infty} q(s) x^{\alpha}[g(s)] \mathrm{d} s \\
& \leq-K l^{\alpha} \int_{t}^{\infty} q(s) \mathrm{d} s, \quad t \geq T .
\end{aligned}
$$

Hence, we have

$$
-\left(r_{1}(t) x^{\prime}(t)\right)^{\prime} \leq-l\left[\frac{1}{r_{2}(t)} \int_{t}^{\infty} K q(s) \mathrm{d} s\right]^{1 / \alpha} .
$$

Integrating the above inequality from $t$ to $\infty$, we obtain

$$
r_{1}(t) x^{\prime}(t) \leq-l K^{1 / \alpha} \int_{t}^{\infty}\left[\frac{1}{r_{2}(s)} \int_{s}^{\infty} q(u) \mathrm{d} u\right]^{1 / \alpha} \mathrm{d} s .
$$

Integrating the last inequality again from $T$ to $t$, we have

$$
\begin{aligned}
& x(t)-x(T) \\
& \leq-l K^{1 / \alpha} \int_{T}^{t} \frac{1}{r_{1}(s)} \int_{s}^{\infty}\left[\frac{1}{r_{2}(u)} \int_{u}^{\infty} q(v) \mathrm{d} v\right]^{1 / \alpha} \mathrm{d} u \mathrm{~d} s .
\end{aligned}
$$

Since condition (18) holds, we obtain $\lim _{t \rightarrow \infty} x(t)=-\infty$, which contradicts $x(t)>0$. Hence, $l=0$. This completes the proof.

\section{Main Results}

In this section, we obtain three new oscillatory criteria for (1) by using the generalized Riccati transformation and integral averaging technique of Philos-type [9]. Let

$$
D=\{(t, s): t \geq s \geq T\} ; \quad D_{0}=\{(t, s): t>s \geq T\} .
$$

A function $H(t, s) \in C^{\prime}(D, R)$ is said to have the property of $P$ and denote $H \in P$ if it satisfies

(i) $H(t, t)=0, t \geq T ; H(t, s)>0,(t, s) \in D_{0}$;

(ii) $\partial H(t, s) / \partial s \leq 0$ and it is continuous.
The following are the main results of this paper.

Theorem 4. Let (8), (18), and $g(t) \geq t$ hold. Assume that there exist $\rho, \varphi \in C^{1}\left(\left[t_{0}, \infty\right),(0, \infty)\right), H(t, s) \in P$, and $m(t, s) \in$ $C(D, R)$, such that

$$
-\frac{\partial H(t, s)}{\partial s}-\frac{R^{*}(s, T)}{\rho(s)} H(t, s)=\frac{m(t, s)}{\rho(s)} \sqrt{H(t, s)},
$$

and for arbitrary $T_{0} \geq T$, one has

$$
\begin{aligned}
& \limsup _{t \rightarrow \infty} \frac{1}{H\left(t, T_{0}\right)} \\
& \quad \times \int_{T_{0}}^{t}\left[H(t, s) Q(s, T)-\frac{m_{-}^{2}(t, s) r_{1}(s)}{4 \alpha \rho(s) R(s, T)}\right] \mathrm{d} s=\infty,
\end{aligned}
$$

where

$$
\begin{gathered}
R(t, T)= \begin{cases}R_{1}(t, T) Q^{1-\alpha}(t), & 0<\alpha \leq 1 ; \\
R_{1}(t, T) R_{2}^{\alpha-1}(t, T), & \alpha \geq 1,\end{cases} \\
R^{*}(t, T)=\rho^{\prime}(t)+\frac{2 \alpha R(t, T)}{r_{1}(t)} r_{2}(t) \varphi(t),
\end{gathered}
$$

$Q(t, T)$

$$
\begin{gathered}
=\rho(t)\left[K q(t)-\left(r_{2}(t) \varphi(t)\right)^{\prime}+\frac{\alpha R(t, T)}{r_{1}(t)}\left(r_{2}(t) \varphi(t)\right)^{2}\right], \\
m_{-}(t, s)=\max \{0,-m(t, s)\} \\
m_{+}(t, s)=\max \{0, m(t, s)\} .
\end{gathered}
$$

Then, every solution $x(t)$ of (1) is oscillatory or converges to zero.

Proof. Assume that (1) has a nonoscillatory solution $x(t)$ on $\left[t_{0}, \infty\right)$. Without loss of generality we may assume that there exists a sufficiently large $t_{1} \geq t_{0}$, such that $x(t)>0, t \geq t_{1}$. By Lemma 1 , we see that $x(t)$ satisfies either case (I) or case (II).

If case (I) holds, then $x^{\prime}(t)>0, t \geq T$. Define the function $W(t)$ by

$$
W(t)=\rho(t) r_{2}(t)\left[\left(\frac{\left(r_{1}(t) x^{\prime}(t)\right)^{\prime}}{x(t)}\right)^{\alpha}+\varphi(t)\right] .
$$

Then,

$$
\begin{aligned}
W^{\prime}(t)= & \frac{\rho^{\prime}(t)}{\rho(t)} W(t)-\rho(t) \frac{q(t) f(x[g(t)])}{x^{\alpha}(t)} \\
& +\rho(t)\left(r_{2}(t) \varphi(t)\right)^{\prime} \\
& -\alpha \rho(t) r_{2}(t)\left[\frac{\left(r_{1}(t) x^{\prime}(t)\right)^{\prime}}{x(t)}\right]^{\alpha} \frac{x^{\prime}(t)}{x(t)} .
\end{aligned}
$$


When $0<\alpha \leq 1$ holds, using (9) and (11), we get

$$
\begin{aligned}
\frac{x^{\prime}(t)}{x(t)}> & \frac{R_{1}(t, T)}{r_{1}(t)} r_{2}(t)\left[\frac{\left(r_{1}(t) x^{\prime}(t)\right)^{\prime}}{x(t)}\right]^{\alpha} \\
& \times\left[\frac{r_{2}^{1 / \alpha}(t)\left(r_{1}(t) x^{\prime}(t)\right)^{\prime}}{x(t)}\right]^{1-\alpha} \\
> & \frac{R_{1}(t, T) Q^{1-\alpha}(t)}{r_{1}(t)} r_{2}(t)\left[\frac{\left(r_{1}(t) x^{\prime}(t)\right)^{\prime}}{x(t)}\right]^{\alpha} .
\end{aligned}
$$

In view of (28) and (29), noting that $g(t) \geq t$, we obtain

$$
\begin{aligned}
W^{\prime}(t) \leq & -K \rho(t) q(t)+\rho(t)\left(r_{2}(t) \varphi(t)\right)^{\prime}+\frac{\rho^{\prime}(t)}{\rho(t)} W(t) \\
& -\alpha \rho(t) \frac{R_{1}(t, T) Q^{1-\alpha}(t)}{r_{1}(t)} \\
& \times\left[r_{2}(t)\left(\frac{\left(r_{1}(t) x^{\prime}(t)\right)^{\prime}}{x(t)}\right)^{\alpha}\right]^{2} .
\end{aligned}
$$

When $\alpha \geq 1$ holds, using (9) and (10), we have

$$
\begin{aligned}
\frac{x^{\prime}(t)}{x(t)}> & \frac{R_{1}(t, T)}{r_{1}(t)} r_{2}(t)\left[\frac{\left(r_{1}(t) x^{\prime}(t)\right)^{\prime}}{x(t)}\right]^{\alpha} \\
& \times\left[\frac{x(t)}{r_{2}^{1 / \alpha}(t)\left(r_{1}(t) x^{\prime}(t)\right)^{\prime}}\right]^{\alpha-1} \\
> & \frac{R_{1}(t, T) R_{2}^{\alpha-1}(t, T)}{r_{1}(t)} r_{2}(t)\left[\frac{\left(r_{1}(t) x^{\prime}(t)\right)^{\prime}}{x(t)}\right]^{\alpha} .
\end{aligned}
$$

In view of (28) and (31), which yields

$$
\begin{aligned}
W^{\prime}(t) \leq & -K \rho(t) q(t)+\rho(t)\left(r_{2}(t) \varphi(t)\right)^{\prime}+\frac{\rho^{\prime}(t)}{\rho(t)} W(t) \\
& -\alpha \rho(t) \frac{R_{1}(t, T) R_{2}^{\alpha-1}(t, T)}{r_{1}(t)} \\
& \times\left[r_{2}(t)\left(\frac{\left(r_{1}(t) x^{\prime}(t)\right)^{\prime}}{x(t)}\right)^{\alpha}\right]^{2} .
\end{aligned}
$$

From (30), (32), and the definition of $R(t, T)$, we get

$$
\begin{aligned}
W^{\prime}(t) \leq & -K \rho(t) q(t)+\rho(t)\left(r_{2}(t) \varphi(t)\right)^{\prime}+\frac{\rho^{\prime}(t)}{\rho(t)} W(t) \\
& -\alpha \rho(t) \frac{R(t, T)}{r_{1}(t)}\left[r_{2}(t)\left(\frac{\left(r_{1}(t) x^{\prime}(t)\right)^{\prime}}{x(t)}\right)^{\alpha}\right]^{2} .
\end{aligned}
$$

By the definition of $W(t)$, we have

$$
\begin{aligned}
& {\left[r_{2}(t)\left(\frac{\left(r_{1}(t) x^{\prime}(t)\right)^{\prime}}{x(t)}\right)^{\alpha}\right]^{2}} \\
& =\left(\frac{W(t)}{\rho(t)}-r_{2}(t) \varphi(t)\right)^{2} \\
& =\frac{1}{\rho^{2}(t)} W^{2}(t)-\frac{2 r_{2}(t) \varphi(t)}{\rho(t)} W(t) \\
& \quad+\left(r_{2}(t) \varphi(t)\right)^{2} .
\end{aligned}
$$

From (33) and (34), noting the definition of $Q(t, T)$ and $R^{*}(t, T)$, we obtain

$$
Q(t, T) \leq-W^{\prime}(t)+\frac{R^{*}(t, T)}{\rho(t)} W(t)-\frac{\alpha R(t, T)}{\rho(t) r_{1}(t)} W^{2}(t) .
$$

Multiplying both sides of (35), with $t$ replaced by $s$, by $H(t, s)$, integrating with respect to $s$ from $T_{0}$ to $t \geq T_{0}$, we get

$$
\begin{aligned}
\int_{T_{0}}^{t} H(t, s) Q(s, T) \mathrm{d} s \leq & -\int_{T_{0}}^{t} H(t, s) W^{\prime}(s) \mathrm{d} s \\
& +\int_{T_{0}}^{t} \frac{H(t, s) R^{*}(s, T)}{\rho(s)} W(s) \mathrm{d} s \\
& -\int_{T_{0}}^{t} \frac{\alpha H(t, s) R(s, T)}{\rho(s) r_{1}(s)} W^{2}(s) \mathrm{d} s .
\end{aligned}
$$

By integrating parts and using $H(t, s) \in P$ and (24), we obtain

$$
\begin{aligned}
& \int_{T_{0}}^{t} H(t, s) Q(s, T) \mathrm{d} s \\
& \leq H\left(t, T_{0}\right) W\left(T_{0}\right)+\int_{T_{0}}^{t} \frac{\partial H(t, s)}{\partial s} W(s) \mathrm{d} s \\
&+\int_{T_{0}}^{t} \frac{H(t, s) R^{*}(s, T)}{\rho(s)} W(s) \mathrm{d} s \\
&-\int_{T_{0}}^{t} \frac{\alpha H(t, s) R(s, T)}{\rho(s) r_{1}(s)} W^{2}(s) \mathrm{d} s \\
&= H\left(t, T_{0}\right) W\left(T_{0}\right) \\
&+\int_{T_{0}}^{t}\left[-\frac{m(t, s) \sqrt{H(t, s)}}{\rho(s)} W(s)\right. \\
&\left.-\frac{\alpha H(t, s) R(s, T)}{\rho(s) r_{1}(s)} W^{2}(s)\right] \mathrm{d} s
\end{aligned}
$$




$$
\begin{aligned}
& \leq H\left(t, T_{0}\right) W\left(T_{0}\right) \\
& +\int_{T_{0}}^{t}\left[\frac{m_{-}(t, s) \sqrt{H(t, s)}}{\rho(s)} W(s)\right. \\
& \left.\quad-\frac{\alpha H(t, s) R(s, T)}{\rho(s) r_{1}(s)} W^{2}(s)\right] \mathrm{d} s .
\end{aligned}
$$

Using averaging technique, we have

$$
\begin{aligned}
& \frac{m_{-}(t, s) \sqrt{H(t, s)}}{\rho(s)} W(s)-\frac{\alpha H(t, s) R(s, T)}{\rho(s) r_{1}(s)} W^{2}(s) \\
& \quad \leq \frac{m_{-}^{2}(t, s) r_{1}(s)}{4 \alpha \rho(s) R(s, T)}
\end{aligned}
$$

Combining (37) and (38), we get

$$
\begin{aligned}
\frac{1}{H\left(t, T_{0}\right)} \int_{T_{0}}^{t}[ & H(t, s) Q(s, T) \\
& \left.\quad-\frac{m_{-}^{2}(t, s) r_{1}(s)}{4 \alpha \rho(s) R(s, T)}\right] \mathrm{d} s \leq W\left(T_{0}\right),
\end{aligned}
$$

which contradicts (25).

If case (II) holds, from (18), by Lemma $3, \lim _{t \rightarrow \infty} x(t)=0$. This completes the proof.

Theorem 5. Let (8), (18), and $g(t) \geq t$ hold. Assuming that there exist $\rho, \varphi \in C^{1}\left(\left[t_{0}, \infty\right),(0, \infty)\right)$ and, for all sufficiently large $T$, there exists a $T_{0} \geq T$, one has

$$
\limsup _{t \rightarrow \infty} \int_{T_{0}}^{t}\left[Q(s, T)-\frac{r_{1}(s) R^{* 2}(s, T)}{4 \alpha \rho(s) R(s, T)}\right] \mathrm{d} s=\infty,
$$

where $R(t, T), Q(t, T)$, and $R^{*}(t, T)$ are defined in Theorem 4. Then every solution $x(t)$ of $(1)$ is oscillatory or converges to zero.

Proof. Assume that (1) has a nonoscillatory solution $x(t)$ on $\left[t_{0}, \infty\right)$. Without loss of generality, we may assume that there exists a sufficiently large $t_{1} \geq t_{0}$, such that $x(t)>0, t \geq t_{1}$. By Lemma 1 , we see that $x(t)$ satisfies either case (I) or case (II).

If case (I) holds, we proceed in the proof of Theorem 4 and get (34). Then, from the definition of $Q(t, T)$ and $R^{*}(t, T)$, we obtain

$$
W^{\prime}(t)<-Q(t, T)+\frac{R^{*}(t, T)}{\rho(t)} W(t)-\frac{\alpha R(t, T)}{\rho(t) r_{1}(t)} W^{2}(t) .
$$

By using the averaging technique, we find that

$$
\frac{R^{*}(t, T)}{\rho(t)} W(t)-\frac{\alpha R(t, T)}{\rho(t) r_{1}(t)} W^{2}(t) \leq \frac{r_{1}(t) R^{* 2}(t, T)}{4 \alpha \rho(t) R(t, T)} .
$$

Hence, we get

$$
W^{\prime}(t)<-\left[Q(t, T)-\frac{r_{1}(t) R^{* 2}(t, T)}{4 \alpha \rho(t) R(t, T)}\right] .
$$

Integrating (43) from $T_{0}$ to $t$, we have

$$
\begin{aligned}
-W\left(T_{0}\right) & \leq W(t)-W\left(T_{0}\right) \\
& \leq-\int_{T_{0}}^{t}\left[Q(s, T)-\frac{r_{1}(s) R^{* 2}(s, T)}{4 \alpha \rho(s) R(s, T)}\right] \mathrm{d} s .
\end{aligned}
$$

It follows that

$$
\int_{T_{0}}^{t}\left[Q(s, T)-\frac{r_{1}(s) R^{* 2}(s, T)}{4 \alpha \rho(s) R(s, T)}\right] \mathrm{d} s \leq W\left(T_{0}\right),
$$

which contradicts (40).

If case (II) holds, from (18), by Lemma $3, \lim _{t \rightarrow \infty} x(t)=0$. This completes the proof.

By applying Theorem 5 with $\rho(t)=t, \varphi(t)=0$, we have the following result.

Corollary 6. Let (8), (18), and $g(t) \geq t$ hold, and for all sufficiently large $T$, there exists a $T_{0} \geq T$; then, one has

$$
\limsup _{t \rightarrow \infty} \int_{T_{0}}^{t}\left[s q(s)-\frac{r_{1}(s)}{4 \alpha K s R(s, T)}\right] \mathrm{d} s=\infty,
$$

where $R(t, T)$ is defined in Theorem 4. Then every solution $x(t)$ of (1) is oscillatory or converges to zero.

Theorem 7. Let (18) and $g(t) \geq t$ hold. Assume that there exist $\rho \in C^{1}\left(\left[t_{0}, \infty\right),(0, \infty)\right), H(t, s) \in P$, and $h(t, s) \in C(D, R)$, such that

$$
\frac{\partial H(t, s)}{\partial s}+\frac{\rho^{\prime}(s)}{\rho(s)} H(t, s)=-\frac{h(t, s)}{\rho(s)} H^{\alpha /(\alpha+1)}(t, s),
$$

and all sufficiently large $T_{0} \geq T$ such that

$$
\begin{aligned}
\limsup _{t \rightarrow \infty} & \frac{1}{H\left(t, T_{0}\right)} \\
\times \int_{T_{0}}^{t} & {[K H(t, s) \rho(s) q(s)} \\
& \left.\quad-\frac{h_{-}^{\alpha+1}(t, s) r_{1}^{\alpha}(s)}{(\alpha+1)^{\alpha+1}\left[\rho(s) R_{1}(s, T)\right]^{\alpha}}\right] \mathrm{d} s=\infty .
\end{aligned}
$$

Then, every solution $x(t)$ of (1) is oscillatory or converges to zero.

Proof. Assume that (1) has a nonoscillatory solution $x(t)$ on $\left[t_{0}, \infty\right)$. Without loss of generality, we may assume that $x(t)$ is a positive solution of (1). By Lemma 1 , we see that $x(t)$ satisfies either case (I) or case (II).

If case (I) holds, then $x^{\prime}(t)>0, t \geq T$. Define the function $W(t)$ by

$$
W(t)=\rho(t) r_{2}(t)\left[\frac{\left(r_{1}(t) x^{\prime}(t)\right)^{\prime}}{x(t)}\right]^{\alpha}
$$


Using (9), we have

$$
\begin{aligned}
W^{\prime}(t)= & -\rho(t) \frac{q(t) f(x[g(t)])}{x^{\alpha}(t)}+\frac{\rho^{\prime}(t)}{\rho(t)} W(t) \\
& -\alpha \rho(t) r_{2}(t)\left[\frac{\left(r_{1}(t) x^{\prime}(t)\right)^{\prime}}{x(t)}\right]^{\alpha} \frac{x^{\prime}(t)}{x(t)} \\
< & -K \rho(t) q(t)+\frac{\rho^{\prime}(t)}{\rho(t)} W(t) \\
& -\frac{\alpha \rho(t) R_{1}(t, T)}{r_{1}(t)} r_{2}^{1+1 / \alpha}(t)\left[\frac{\left(r_{1}(t) x^{\prime}(t)\right)^{\prime}}{x(t)}\right]^{\alpha+1} .
\end{aligned}
$$

Hence, by the definition of $W(t)$, we have

$$
\begin{aligned}
W^{\prime}(t) \leq & -K \rho(t) q(t)+\frac{\rho^{\prime}(t)}{\rho(t)} W(t) \\
& -\frac{\alpha R_{1}(t, T)}{\rho^{1 / \alpha}(t) r_{1}(t)} W^{1+1 / \alpha}(t) .
\end{aligned}
$$

Multiplying both sides of (51), with $t$ replaced by $s$, by $H(t, s)$ and integrating with respect to $s$ from $T_{0} \geq T$ to $t \geq T_{0}$, we get

$$
\begin{aligned}
\int_{T_{0}}^{t} K H(t, s) \rho(s) q(s) \mathrm{d} s \\
\leq-\int_{T_{0}}^{t} H(t, s) W^{\prime}(s) \mathrm{d} s \\
\quad+\int_{T_{0}}^{t} H(t, s) \frac{\rho^{\prime}(s)}{\rho(s)} W(s) \mathrm{d} s \\
\quad-\int_{T_{0}}^{t} H(t, s) \frac{\alpha R_{1}(s, T)}{\rho^{1 / \alpha}(s) r_{1}(s)} W^{1+1 / \alpha}(s) \mathrm{d} s .
\end{aligned}
$$

Integrating by parts and using (47), which yields

$$
\begin{aligned}
& \int_{T_{0}}^{t} K H(t, s) \rho(s) q(s) \mathrm{d} s \\
& \leq H\left(t, T_{0}\right) W\left(T_{0}\right)+\int_{T_{0}}^{t} \frac{\partial H(t, s)}{\partial s} W(s) \mathrm{d} s \\
&+\int_{T_{0}}^{t} H(t, s) \frac{\rho^{\prime}(s)}{\rho(s)} W(s) \mathrm{d} s \\
&-\int_{T_{0}}^{t} H(t, s) \frac{\alpha R_{1}(s, T)}{\rho^{1 / \alpha}(s) r_{1}(s)} W^{1+1 / \alpha}(s) \mathrm{d} s \\
& \leq H\left(t, T_{0}\right) W\left(T_{0}\right) \\
&+\int_{T_{0}}^{t}\left[-\frac{h(t, s) H^{\alpha /(\alpha+1)}(t, s)}{\rho(s)} W(s)\right. \\
&\left.-H(t, s) \frac{\alpha R_{1}(s, T)}{\rho^{1 / \alpha}(s) r_{1}(s)} W^{1+1 / \alpha}(s)\right] \mathrm{d} s
\end{aligned}
$$

$$
\begin{aligned}
& \leq H\left(t, T_{0}\right) W\left(T_{0}\right) \\
& +\int_{T_{0}}^{t}\left[\frac{h_{-}(t, s) H^{\alpha /(\alpha+1)}(t, s)}{\rho(s)} W(s)\right. \\
& \left.\quad-H(t, s) \frac{\alpha R_{1}(s, T)}{\rho^{1 / \alpha}(s) r_{1}(s)} W^{1+1 / \alpha}(s)\right] \mathrm{d} s .
\end{aligned}
$$

Define $A$ and $B$ as follows:

$$
\begin{gathered}
A^{\lambda}=\frac{\alpha H(t, s) R_{1}(s, T)}{\rho^{1 / \alpha}(s) r_{1}(s)} W^{\lambda}(s), \\
B^{\lambda-1}=\frac{h_{-}(t, s) r_{1}^{1 / \lambda}(s)}{\lambda\left[\alpha \rho(s) R_{1}(s, T)\right]^{1 / \lambda}},
\end{gathered}
$$

where $\lambda=(\alpha+1) / \alpha>1, A \geq 0$, and $B \geq 0$. Using the inequality [10, Theorem 41]

$$
\lambda A B^{\lambda-1}-A^{\lambda} \leq(\lambda-1) B^{\lambda},
$$

we obtain

$$
\begin{aligned}
& \frac{h_{-}(t, s) H^{1 / \lambda}(t, s)}{\rho(s)} W(s)-\frac{\alpha H(t, s) R_{1}(s, T)}{\rho^{\lambda-1}(s) r_{1}(s)} W^{\lambda}(s) \\
& \leq \frac{h_{-}^{\alpha+1}(t, s) r_{1}^{\alpha}(s)}{(\alpha+1)^{\alpha+1}\left[\rho(s) R_{1}(s, T)\right]^{\alpha}} .
\end{aligned}
$$

Combining (53) and (56), we get

$$
\begin{aligned}
\frac{1}{H\left(t, T_{0}\right)} \int_{T_{0}}^{t}[ & K H(t, s) \rho(s) q(s) \\
& \left.\quad-\frac{h_{-}^{\alpha+1}(t, s) r_{1}^{\alpha}(s)}{(\alpha+1)^{\alpha+1}\left[\rho(s) R_{1}(s, T)\right]^{\alpha}}\right] \mathrm{d} s \leq W\left(T_{0}\right),
\end{aligned}
$$

which contradicts (48).

If case (II) holds, from (18), by Lemma $3, \lim _{t \rightarrow \infty} x(t)=$ 0 . This completes the proof.

Remark 8. If we let $\rho(t)=1, \varphi(t)=0$ in Theorem 4 and the function $m(t, s)$ is $Q(t, s)$ of Theorem 3.3 in [5], then condition (25) is (3.12) in [5]. Therefore, the result of $\alpha=1$ in [5] is generalized to the case that $\alpha$ is the ratio of positive odd integers. If we let $\rho(t)=1$ in Theorem 7 , the function $h_{-}^{\alpha+1}(t, s)$ is $Q^{\alpha+1}(t, s) / H^{\alpha}(t, s)$ of Theorem 3.4 in [3], which condition (48) is converted to (3.19) in [3]. Then, the result of (1.3) in [3] is generalized to the one of (1) in this paper.

Example 9. Consider the three-order differential equation

$$
\left(\left[\left(\frac{1}{t} x^{\prime}(t)\right)^{\prime}\right]^{3 / 5}\right)^{\prime}+\frac{1}{t^{2}} x^{3 / 5}(t)=0, \quad t \in[1,+\infty),
$$

where

$$
\begin{aligned}
r_{1}(t) & =\frac{1}{t}, & r_{2}(t)=1, & q(t)=\frac{1}{t^{2}} \\
f(u) & =u^{3 / 5}, & g(t)=t, & \alpha=\frac{3}{5} .
\end{aligned}
$$


Conditions $\left(A_{1}\right),\left(A_{2}\right)$, and $\left(A_{3}\right)$ are clearly satisfied. It is easy to find that (8) and (18) hold. Let $\rho(t)=t, \varphi(t)=1 / t$. Here

$$
\begin{aligned}
& R_{1}(t, T)=\int_{T}^{t}\left(\frac{1}{r_{2}(s)}\right)^{1 / \alpha} \mathrm{d} s=\int_{T}^{t} \mathrm{~d} s=t-T \\
& Q(t)=\left(K \int_{t}^{\infty} q(s) \mathrm{d} s\right)^{1 / \alpha}=\left(\int_{t}^{\infty} \frac{1}{s^{2}} \mathrm{~d} s\right)^{5 / 3}=t^{-5 / 3} \\
& R(t, T)=R_{1}(t, T) Q^{1-\alpha}(t)=(t-T) t^{-2 / 3} \\
& R^{*}(t, T)=\rho^{\prime}(t)+\frac{2 \alpha R(t, T)}{r_{1}(t)} r_{2}(t) \varphi(t) \rho(t) \\
&=1+\frac{6}{5}(t-T) t^{1 / 3}, \\
& Q(t, T)=\rho(t)\left[K q(t)-\left(r_{2}(t) \varphi(t)\right)^{\prime}\right. \\
&\left.+\frac{\alpha R(t, T)}{r_{1}(t)}\left(r_{2}(t) \varphi(t)\right)^{2}\right] \\
&=\frac{2}{t}+\frac{3}{5}(t-T) t^{-2 / 3}
\end{aligned}
$$

From Theorem 5, we have

$$
\begin{aligned}
& \limsup _{t \rightarrow \infty} \int_{1}^{t}\left[Q(s, T)-\frac{r_{1}(s) R^{* 2}(s, T)}{4 \alpha \rho(s) R(s, T)}\right] \mathrm{d} s \\
& \quad=\limsup _{t \rightarrow \infty} \int_{1}^{t}\left[\frac{1}{s}-\frac{5}{12} \frac{1}{(s-T) s^{4 / 3}}\right] \mathrm{d} s=\infty,
\end{aligned}
$$

so (40) is satisfied. Hence, by Theorem 5 , every solution $x(t)$ of (58) is oscillatory or converges to zero.

\section{Conflict of Interests}

The authors declare that there is no conflict of interests regarding the publication of this paper.

\section{Acknowledgments}

This work is supported by the Natural Science Foundation of Shandong Province of China (ZR2013AM003), the Development Program in Science and Technology of Shandong Province of China (2010GWZ20401), and the Science Foundation of Binzhou University (BZXYKJ0810).

\section{References}

[1] G. S. Ladde, V. Lakshmikantham, and B. G. Zhang, Oscillation Theory of Differential Equations with Deviating Arguments, vol. 110, Marcel Dekker, New York, NY, USA, 1987.

[2] N. Parhi and S. Padhi, "Asymptotic behaviour of solutions of third order delay-differential equations," Indian Journal of Pure and Applied Mathematics, vol. 33, no. 10, pp. 1609-1620, 2002.

[3] B. Baculíková, E. M. Elabbasy, S. H. Saker, and J. Džurina, "Oscillation criteria for third-order nonlinear differential equations," Mathematica Slovaca, vol. 58, no. 2, pp. 201-220, 2008.
[4] I. Mojsej, "Asymptotic properties of solutions of third-order nonlinear differential equations with deviating argument," Nonlinear Analysis. Theory, Methods \& Applications, vol. 68, no. 11, pp. 3581-3591, 2008.

[5] S. H. Saker, "Oscillation criteria of third-order nonlinear delay differential equations," Mathematica Slovaca, vol. 56, no. 4, pp. 433-450, 2006.

[6] B. Baculíková and J. Džurina, "Oscillation of third-order functional differential equations," Electronic Journal of Qualitative Theory of Differential Equations, vol. 43, pp. 1-10, 2010.

[7] B. Baculíková and J. Džurina, "Oscillation of third-order nonlinear differential equations," Applied Mathematics Letters, vol. 24, no. 4, pp. 466-470, 2011.

[8] S. R. Grace, R. P. Agarwal, R. Pavani, and E. Thandapani, "On the oscillation of certain third order nonlinear functional differential equations," Applied Mathematics and Computation, vol. 202, no. 1, pp. 102-112, 2008.

[9] C. G. Philos, "Oscillation theorems for linear differential equations of second order," Archiv der Mathematik, vol. 53, no. 5, pp. 482-492, 1989.

[10] G. H. Hardy, J. E. Littlewood, and G. Pólya, Inequalities, Cambridge University Press, Cambridge, UK, 2nd edition, 1988. 


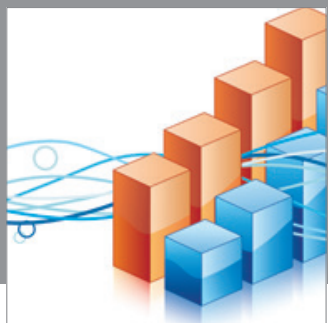

Advances in

Operations Research

mansans

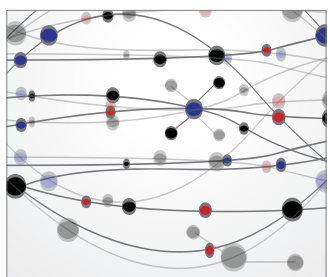

The Scientific World Journal
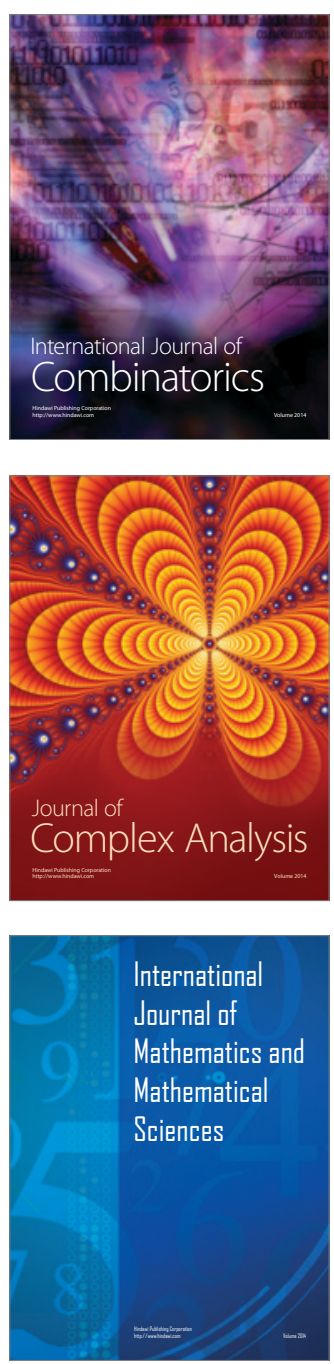
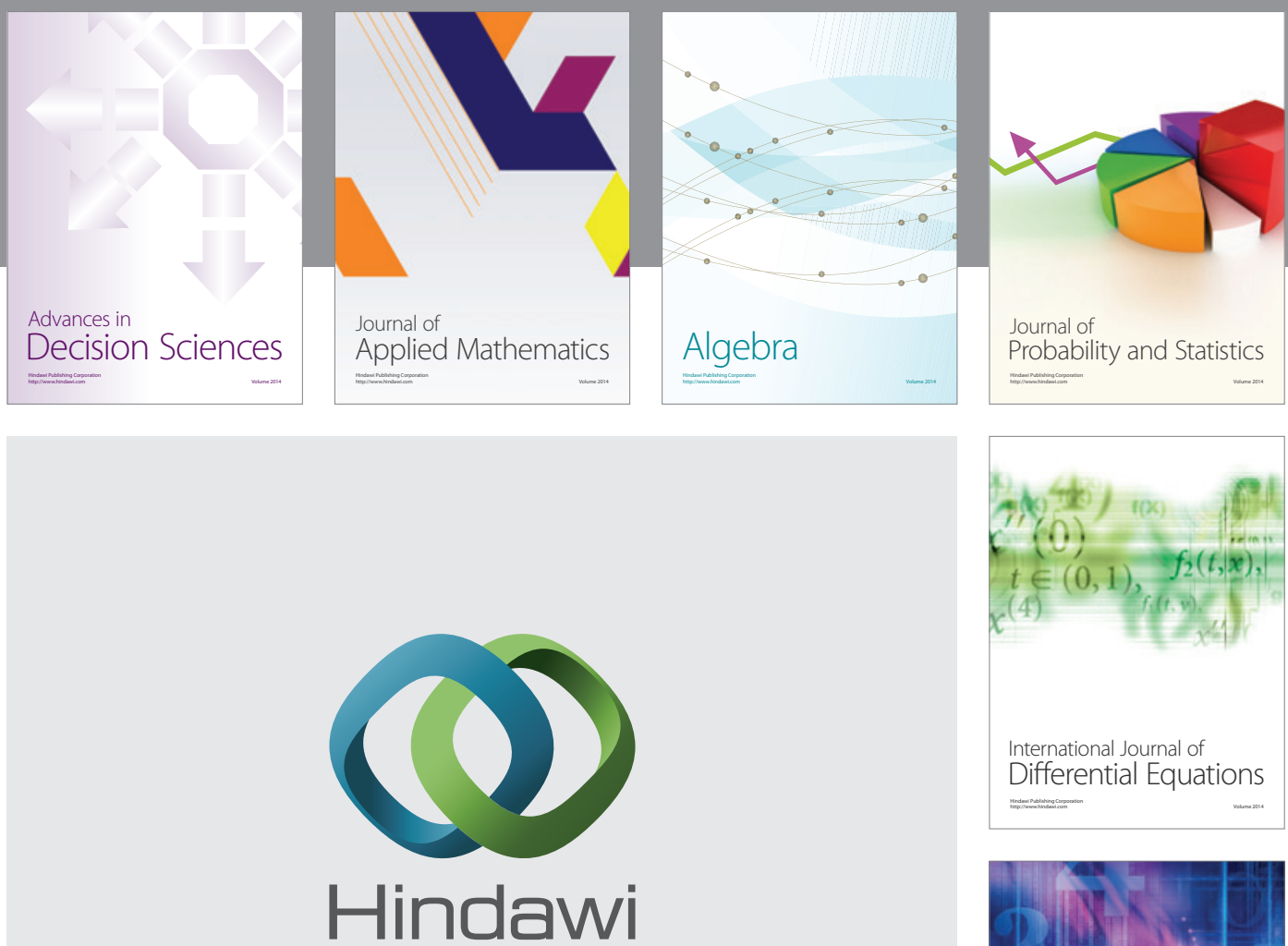

Submit your manuscripts at http://www.hindawi.com
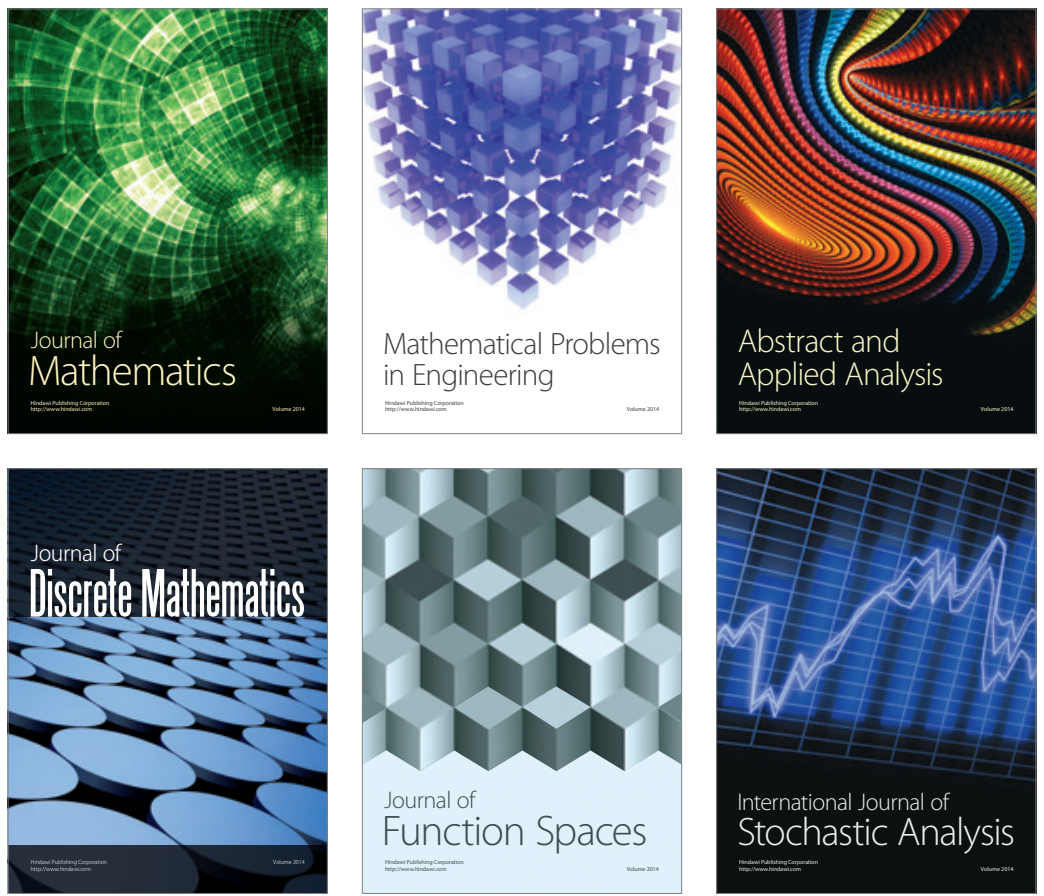

Journal of

Function Spaces

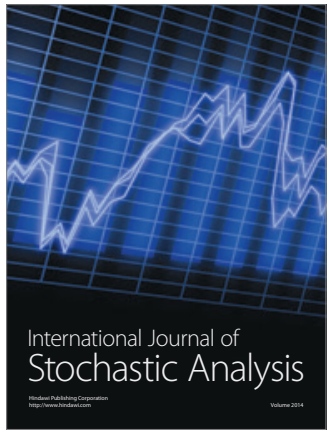

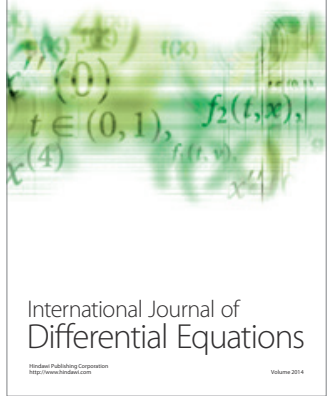
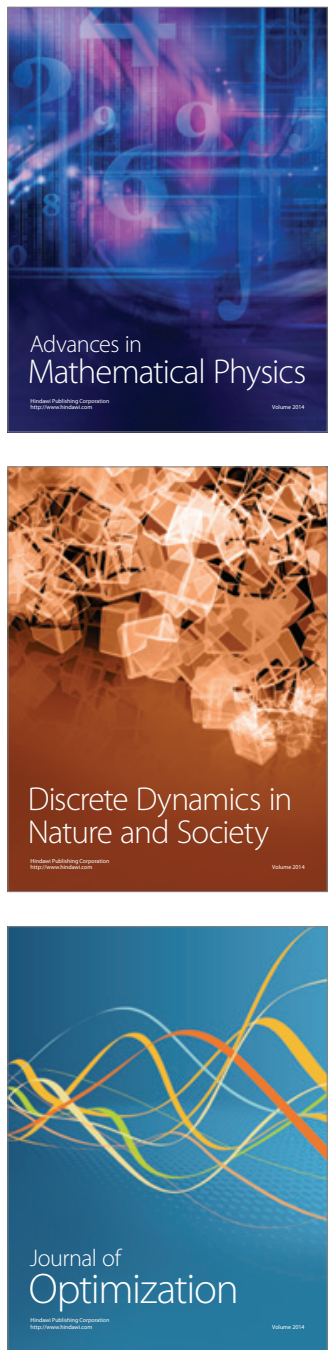